\title{
Recent Endoscopic Ultrasound-Related Publications With Potential to Influence Clinical Practice
}

\author{
Atul Gawande ${ }^{1}$ Shrikant Mukewar ${ }^{1} \quad$ Ravi Daswani $^{1} \quad$ Bhushan Bhaware $^{1}$ Saurabh Mukewar ${ }^{1}$ \\ 1 Department of Gastroenterology, Midas Multispeciality Hospital, \\ Nagpur, Maharashtra, India

\begin{abstract}
Address for correspondence Saurabh Mukewar, MD, Department of Gastroenterology, Midas Multispeciality Hospital, Nagpur, Maharashtra 440010, India (e-mail: saurabhmukewar@gmail.com).
\end{abstract}

J Digest Endosc 2021;12:221-228.

\author{
Abstract \\ Keywords \\ - endoscopic \\ ultrasound \\ - gall bladder drainage \\ - acute necrotizing \\ pancreatitis \\ - lumen-apposing \\ metal stent \\ - portal hypertension \\ - solid pancreatic \\ lesion
}

The field of endoscopic ultrasound (EUS) has evolved significantly over the last two decades from being a tool of only diagnostic purpose to tissue acquisition and now therapeutic potential. There have been several important publications in the field of EUS in the last few years, which had a major impact in the clinical management of various gastrointestinal disorders. In this review, we discuss four such articles that in our opinion will significantly impact the role of EUS in treating various conditions. The first article is a randomized controlled trial comparing EUS-guided gall bladder drainage with percutaneous gall bladder drainage for high-risk acute cholecystitis. The second article is a randomized controlled trial comparing EUS versus minimally invasive surgery for necrotizing pancreatitis. The third article is a novel human study of EUS-guided portal pressure measurement in patients with portal hypertension. The last article is also a randomized controlled trial evaluating the role of rapid on-site evaluation for EUS-guided fine needle biopsy in solid pancreatic lesions.

\section{Introduction}

The field of endoscopic ultrasound (EUS) has evolved significantly over the last three decades. From being a tool for diagnostic evaluation with limited indications, EUS now has not only diagnostic potential but also several therapeutic indications such as drainage of perienteral fluid collections, drainage of pancreatic and bile duct, and creation of enteroenterostomy in obstruction and hemostasis in bleeding from pseudoaneurysms and varices. This has been possible due to not only development and improvement in EUS scopes and image quality but also advancements in devices and accessories for EUS, enabling the field of interventional EUS to blossom. In this review, we discuss four recent articles that we feel have had a significant impact in the field of EUS.

\section{Endosonography-Guided Drainage of Gallbladder in High-Surgical-Risk Patients with Acute Cholecystitis}

The gold standard of treatment for acute cholecystitis is laparoscopic cholecystectomy. ${ }^{1}$ However, in the elderly and in high-risk patients for surgery, minimally invasive options are desired. Traditionally, percutaneous gall bladder drainage (PT-GBD) is used for surgically unfit or high-risk patients. The adverse events associated with PT-GBD are up to $14 \%$, including pneumothorax, subcapsular hematoma, bile leak, pain, and misplacement or migration of catheter requiring repeated procedure. ${ }^{2-4}$ Also, as the bile is drained outside the body, it increases stress on the liver to produce more bile to maintain bile acid pool. PT-GBD is contraindicated in thrombocytopenia, coagulopathy, large ascites, and Chilaiditi's

(C) 2022. Society of Gastrointestinal Endoscopy of India. All rights reserved.

This is an open access article published by Thieme under the terms of the Creative Commons Attribution-NonDerivative-NonCommercial-License, permitting copying and reproduction so long as the original work is given appropriate credit. Contents may not be used for commercial purposes, or adapted, remixed, transformed or built upon. (https://creativecommons.org/ licenses/by-nc-nd/4.0/)

Thieme Medical and Scientific Publishers Pvt. Ltd., A-12, 2nd Floor, Sector 2, Noida-201301 UP, India 


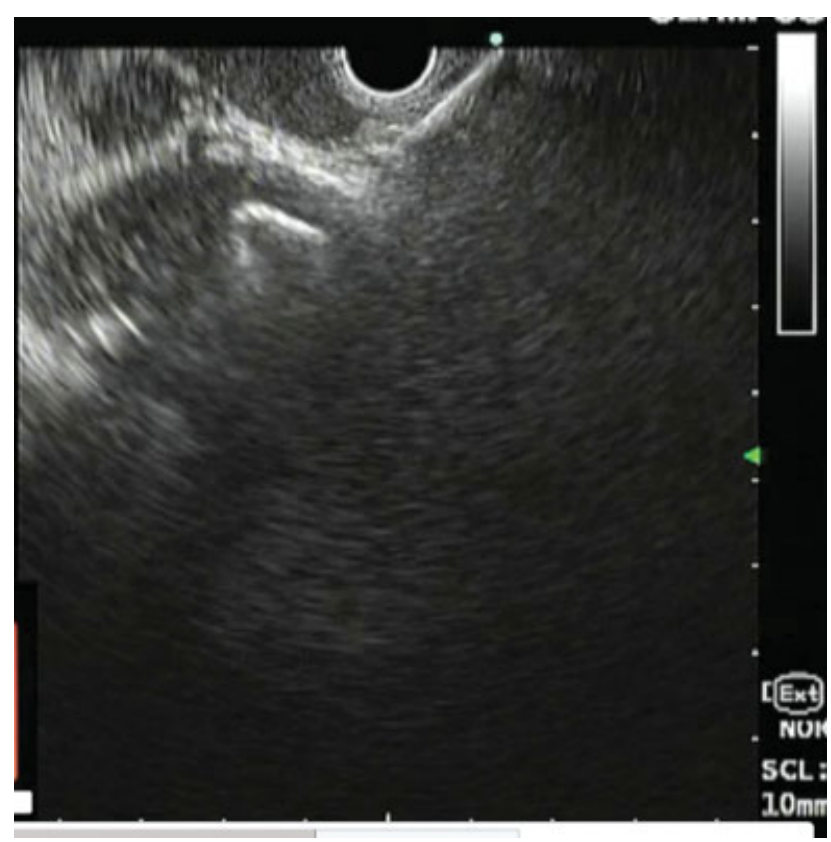

Fig. 1 EUS view of lumen-apposing metal stent being deployed in the gallbladder across the gastric wall in a patient with acute cholecystitis.

syndrome. ${ }^{5}$ PT-GBD offers a temporary solution and eventually surgery is needed, once the patient is considered fit to undergo surgery. If surgical risk persists, the PT-GBD catheter needs to be kept long term, which can be quite inconvenient, and problems such as tube dislodgement can occur. Recently, EUS-guided gallbladder drainage (EUS-GBD) has gained attention for internally draining the gallbladder in high-risk patients (-Fig. $\mathbf{1}$ and - Fig. 2). This not only is a temporizing measure but also can provide a convenient option for long-term internal drainage in patients in whom surgical risk persists. Multiple retrospective comparative

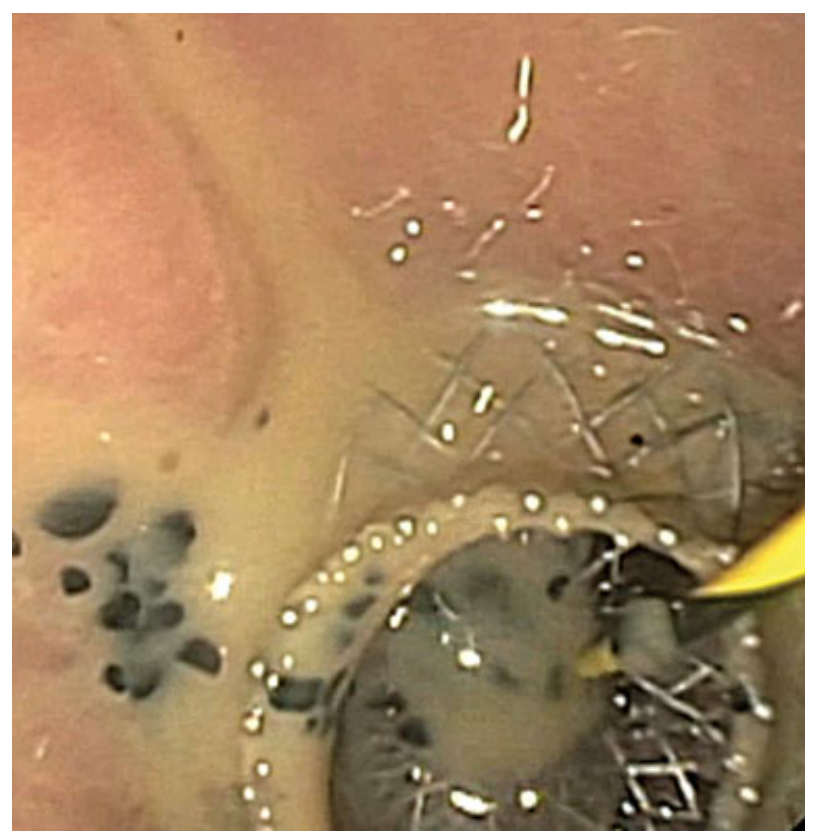

Fig. 2 Endoscopic image of lumen-apposing metal stent draining pus and stones. studies showed that EUS-GBD is similar to PT-GBD in terms of technical and clinical success, and EUS-GBD is associated with better outcomes in terms of reinterventions, hospital stay, and pain scores. ${ }^{6-8}$

Teoh et $\mathrm{al}^{9}$ conducted a multicenter prospective randomized superiority trial comparing EUS-GBD with PT-GBD for high-risk acute cholecystitis patients in five centers in China and Japan. In this study, 665 patients were screened and majority of them, almost $80 \%$ (545 patients), were candidates for cholecystectomy and were not considered for the trial. Additionally, 40 other patients were excluded as they did not fit the inclusion criteria. Finally, 80 patients were randomized (40 in EUS-GBD and 40 in PT-GBD group). Expert endosonographers (more than 25 EUS-GBD experience) performed EUSGBD via transgastric or transduodenal route. Technique was either direct placement of the lumen-apposing metal stent (LAMS) via EUS or conventional technique with EUS guided advancement of a 19G Fine Needle Aspiration (FNA) needle, guidewire coiling followed by stent deployment. The procedure was performed under conscious sedation. On follow-up, the stent and stones were removed and replaced with plastic stents. PT-GBD was performed by experienced interventional radiologists ( $>50$ PT-GBD experience) via transhepatic (preferred) or transperitoneal route. Patients in PT-GBD group underwent tube cholecystogram after 1 month, and if the cystic duct was patent, then cholecystostomy tube was clamped or removed. And if the cystic duct was not patent, then the tube was placed in situ as it is for long-term cholecystostomy drainage. The primary outcome measurement was the cumulative rate of adverse events in 1 year. There was no difference between the groups on baseline demographics.

Those undergoing EUS-GBD, compared with PT-GBD, had significantly reduced 30 -day adverse events ( 12.8 vs. $47.5 \%)$ and 1 -year adverse events (25.6 vs. $77.5 \%$ ). The majority of the 30-day and 1-year adverse events in the PT-GBD group were due to tube dislodgements, thus resulting in higher risk of recurrence of cholecystitis in the PT-GBD arm. However, the 30 -day mortality (7.7\% in EUS-GBD vs. $10 \%$ in PT-GBD) was not significantly different. There were no differences in technical success (97.4\% in EUS-GBD vs. $100 \%$ in PT-GBD) and clinical success (92.3\% in EUS-GBD vs. $92.5 \%$ in PT-GBD) between the two groups. In one patient in the EUS-GBD arm, the procedure could not be performed due to inability to find a transgastric or transduodenal window. Analgesic requirements were less in the EUS-GBD cohort. Rates of reinterventions were also less for the EUS-GBD cohort. The procedural time and hospital stay were not significantly different. None of the patients in either group underwent cholecystectomy. Limitations of this study was that the follow-up of the study was short. Thus, long-term complications, if any, of this novel procedure are not known. Costeffectiveness of EUS-GBD was also not determined in this study.

Results from this study as well as prior study consistently suggest superior outcomes of EUS-GBD over PT-GBD. ${ }^{7,10}$ The current study is a randomized trial compared with prior retrospective studies. The results are expected as EUS-GBD 


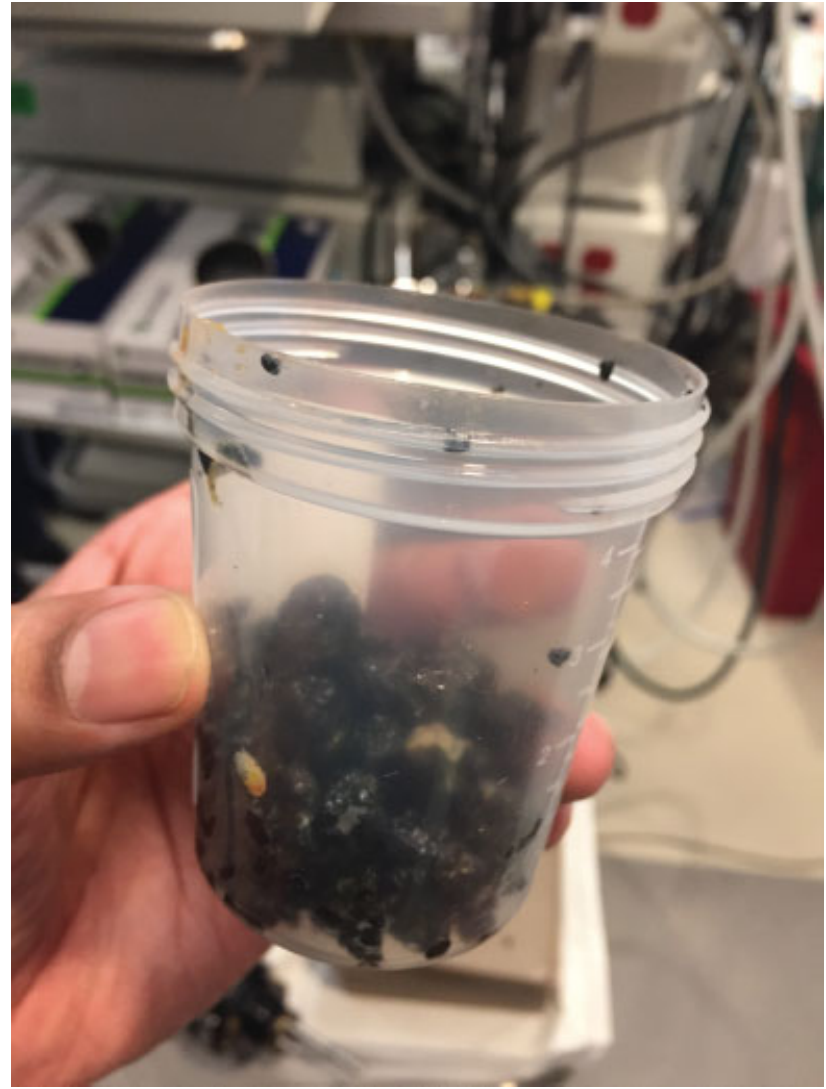

Fig. 3 Gall stones removed after EUS-guided gall bladder drainage with a lumen-apposing metal stent procedure.

in experienced hands is a safe and quick procedure, offering internal drainage through natural orifices of an infected closed cavity. Similar findings have been seen for other conditions such as infected walled-off pancreatic necrosis and postsurgical collections. ${ }^{11,12}$ EUS-GBD also permits removal of stones from the gallbladder ( - Fig. 3). The cost of EUS-GBD along with the need for LAMS can be a challenge, especially in the developing world, limiting its applicability. In patients who are likely to be candidates for cholecystectomy in future, perhaps PT-GBD is a better option since no internal fistulas are created and the surgeons are able to remove the gallbladder easily. However, one study has shown no difference in rates of technical success, adverse events, or conversion to open cholecystectomy in those undergoing surgery following EUSGBD versus PT-GBD. ${ }^{13}$ The challenge with PT-GBD lies in the elderly patients and those who will likely remain high risk for cholecystectomy. As seen in this study and prior studies, ${ }^{2-4}$ such patients can have several problems related to the tube, which can get dislodged or blocked, resulting in recurrent cholecystitis and reinterventions. Also, the procedure leads to more pain and there is the added inconvenience of tube, which can affect the quality of life.

In conclusion, EUS-GBD appears superior to PT-GBD for high-risk acute cholecystitis patients. Whether this should be performed in patients who will eventually become candidates for cholecystectomy is unclear. We have performed EUS-GBD in other indications beyond acute cholecystitis such as high-risk surgery patients with biliary colic. Also,
EUS-GBD has been performed for drainage of malignant biliary obstruction after failed endoscopic retrograde cholangiopancreatography and EUS-guided bile duct drainage with clinical and technical success rates of 92.6 and $100 \%$, respectively. ${ }^{14}$ Further studies expanding the indications of this promising procedure in conditions such as high-risk cholecystectomy for biliary colic or acute biliary pancreatitis or even in surgical candidates who would prefer nonsurgical alternative are required.

\section{An Endoscopic Transluminal Approach Compared with Minimally Invasive Surgery in Patients with Acute Necrotizing Pancreatitis}

Necrosis occurs in $20 \%$ of patients with acute pancreatitis with mortality rate of 8 to $39 \%{ }^{15}$ Secondary infection of necrosis can lead to sepsis and organ failure. ${ }^{16}$ Management of necrotizing pancreatitis has evolved from open surgical necrosectomy to minimally invasive approaches (percutaneous, endoscopic, and/or surgical) due to higher complications such as organ failure and endocrine/exocrine insufficiency from open surgical approach. ${ }^{17}$ An initial small pilot randomized trial (PENGUIN trial) comparing minimally invasive surgical approach with endoscopic approach showed superior outcomes with endoscopic approach with lower inflammation (lower interleukin 6 [IL-6]) levels and lesser complications such as new-onset organ failure and pancreatic fistula. ${ }^{18}$ The authors followed this with a larger study (TENSION trial) comparing the two approaches and showed no difference in mortality, but lesser fistulas and hospital stay with endoscopic approach. ${ }^{19}$

Bang et $a l,{ }^{11}$ in their single-center randomized trial, compared endoscopic transluminal approach $(n=34)$ with minimal invasive surgery $(n=32)$ for infected necrotizing pancreatitis. Indications for intervention were consistent with recent guidelines. Surgical approach included either laparoscopic cystogastrostomy with necrosectomy $(n=26)$ or video-assisted retroperitoneal debridement (VARD) $(n=6)$. Endoscopic approach included endosonographyguided transgastric or transduodenal drainage with single/multiple plastic stents $(n=18)$ or metal stents $(n=16)$ (-Fig. 4) with additional percutaneous drainage if the collection extended to flanks. If the collections were unilocular with size larger than $60 \mathrm{~mm}$ but smaller than $80 \mathrm{~mm}$, then it was treated by single-tract transmural cystogastrostomy/duodenostomy (single-gate technique). If the collections were larger than $80 \mathrm{~mm}$ in size or extended to the flanks, then it was treated by creation of multiple transmural tract (multigate technique). Necrosectomy (-Fig. 5) was performed in almost the entire surgery group (96.9\%) but only in select patients in endoscopy group (32.4\%) who did not have improvement on drainage alone. Majority of the patients randomized were assigned to one of the two groups -only four excluded (two for protocol violation and two for resolution of symptoms following percutaneous drainage). Both groups were well matched with no significant difference in baseline characteristics. 


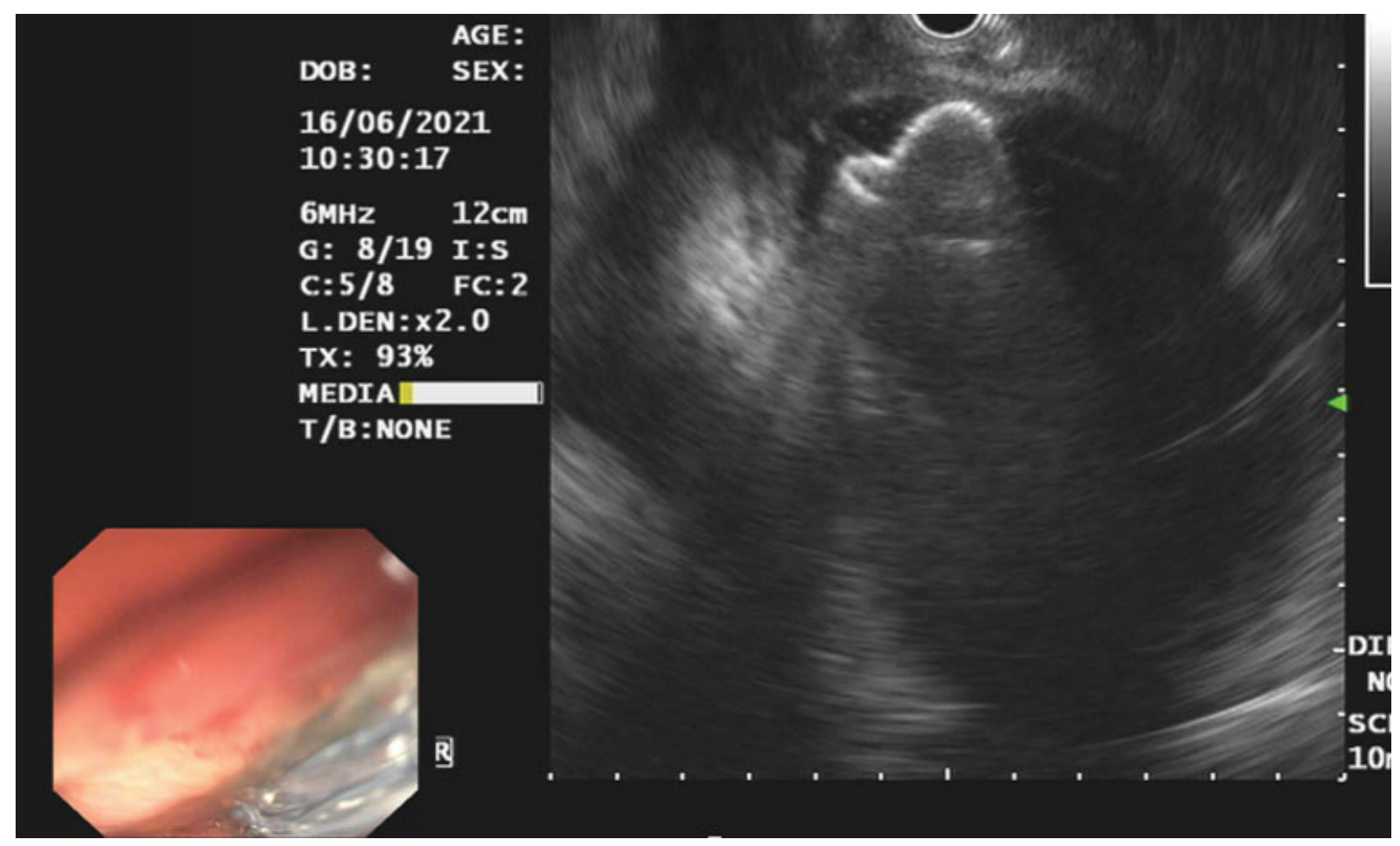

Fig. 4 EUS-guided lumen-apposing metal stent for walled-off pancreatic necrosis.

The composite primary end point of major complications or death occurred in significantly fewer number of patients in the endoscopy cohort: $4(11.8 \%)$ versus $13(40.6 \%)$ in the surgical cohort. The difference was mainly due to enteral or pancreatic-cutaneous fistulas in the surgical cohort (28.6 vs. $0 \%$ ). On Cox's proportional hazard analysis, undergoing surgery and high Acute Physiology and Chronic Health Evaluation (APACHE) scores were the only factors independently associated with the outcome of major complications or death. The mean number of major complications per patient was significantly higher for surgery compared with endoscopy ( 0.69 vs. 0.15 ). There was no difference in procedure-related adverse events but disease-related adverse events such as pain and infection were more in the surgical group compared with endoscopy at 6-month follow-up.

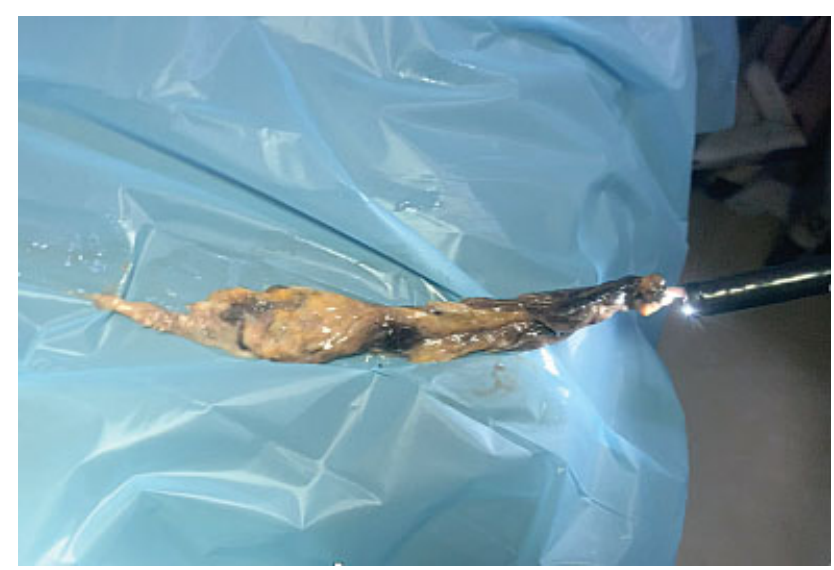

Fig. 5 Pancreatic necrosis removed with endoscope.
Procedure duration was shorter in the endoscopy cohort. Necrosectomy was only needed in $32.4 \%$ in the endoscopy arm compared with $96.9 \%$ in the surgery arm. The endoscopy cohort also had lower rate of systemic inflammatory response syndrome (SIRS), early resolution of pre-existing SIRS, and fewer patients with new-onset SIRS. In addition, the length of intensive care unit and hospital stay was shorter for the endoscopy group. Patients in endoscopy group had lower readmission rate and higher quality of life. The mean total of direct and indirect medical costs per patient during admission and up to 6-month follow-up was also higher for the surgery group.

SIRS is a driver for complications in patients with pancreatitis. ${ }^{20}$ In the current study, SIRS reduced following endoscopic approach but increased following surgical approach. The likely reason is that in surgical approach the cavity is accessed via percutaneous route, with spillage of secretions resulting in tissue inflammation/necrosis. The rigid surgical instruments do not permit access to difficult locations and cause trauma to tissues and cavity walls. On the contrary, endoscopic approach utilizes natural orifices and flexible endoscopes permit access to difficult locations, permitting gentle debridement of necrotic tissue under direct visualization, thus leading to less trauma to body tissues, lower inflammation, and SIRS.

Surgical procedures require general anesthesia, which itself is proinflammatory for such critically ill patients, and the procedures are of prolonged duration, as shown in the current study. ${ }^{21}$ In comparison, endoscopic procedures are shorter and can be performed under conscious sedation. The study also shows more disease-related adverse events such as pain and infection in the surgery arm. This is 
expected since a percutaneous route is required for surgery, resulting in more pain scores. These adverse events ultimately lead to lower quality of life and higher costs for management of patients. The study was conducted in a single tertiary center in the United States, limiting its applicability to the Indian population, where patients are possibly more malnourished and prone to more infections. Also, different endoprostheses were used in different patients, leading to heterogeneity.

This study shows convincing evidence to support endoscopic approach for management of necrotizing pancreatitis (-Fig. 4 and -Fig. 5). Overall, reduced number of complications with lower cost and better quality of life were seen in the endoscopy cohort. The prior TENSION trial showed a trend toward better outcomes with endoscopy but the results were not as significant as this study. This is likely due to differences in study methodology-only patients undergoing surgery (both laparoscopic approach and VARD) were included in this trial versus in TENSION trial where all patients undergoing percutaneous drainage without surgery were also considered. Patients were much sicker in this study with higher APACHE scores and higher American Society of Anesthesiologists (ASA) grades compared with TENSION trial. Almost half the patients in the endoscopy arm underwent LAMSs in this study, which are shown to have superior outcomes to plastic stents. In comparison, in TENSION trial only plastic stents were used.

In conclusion, endoscopic transmural approach appears to have superior outcomes to minimally invasive surgery with lower major complications and better quality of life with lower costs.

\section{Endosonography-Guided Portal Pressure Gradient Measurement}

Portal hypertension (PH) is a major complication of liver cirrhosis. Patients with $\mathrm{PH}$ are at risk of developing gastroesophageal varices and related bleeding, ascites, hepatorenal syndrome, and hepatic encephalopathy. ${ }^{22}$ Although not performed routinely, quantitative measurement of $\mathrm{PH}$ has therapeutic and prognostic implications. ${ }^{23-25}$

Portal pressure gradient (PPG) is calculated by subtracting hepatic vein (HV) pressure from portal vein (PV) pressure. Ideally, direct pressure measurement should be taken from HV and PV. However, as this is technically challenging, interventional radiology (IR)-guided indirect measurement of PV pressure by wedged hepatic venous pressure (WHVP) is performed. Hepatic venous pressure gradient (HVPG) is the difference between WHVP (i.e., estimated portal venous pressure) and HV pressure. $\mathrm{PH}$ is defined as HVPG $>5 \mathrm{~mm}$ $\mathrm{Hg}$, while clinically significant $\mathrm{PH}$ (CSPH) is defined as HVPG $>10 \mathrm{~mm} \mathrm{Hg}$. The risk of variceal bleeding is dramatically lowered if HVPG is reduced by $20 \%$ from baseline or an absolute value $<12 \mathrm{~mm} \mathrm{Hg}$ is achieved. ${ }^{26-28}$ Thus, HVPG monitoring can guide pharmacologic prophylaxis in patients with varices. Also, $\mathrm{PH}$ is an independent factor for survival in patients with cirrhosis. ${ }^{29}$ EUS-guided direct portal pressure measurement using a $25-\mathrm{G}$ needle has been performed in animal models successfully. It has also shown high accuracy and strong correlation of pressure values obtained by conventional (IR- Interventional Radiology) methods in animal model. ${ }^{30}$ Also, Zhang et al had demonstrated that EUSguided PPG measurement not only is safe and feasible but also has strong correlation with HVPG measurement in human subjects. ${ }^{31}$

Huang et $\mathrm{al}^{32}$ conducted the first human study of direct portal and hepatic venous pressure measurement via EUS using a $25-G$ needle attached to a novel compact manometer in 28 patients. Patients between the age of 18 and 75 years with a history of liver disease or suspected cirrhosis were considered for PPG measurement. Exclusion criteria included pregnancy, significant bleeding risk (international normalized ratio $>1.5$, platelet count $<50 \times 10^{9} / \mathrm{L}$ ), active gastrointestinal (GI) bleeding, and postsinusoidal PH. Patients were deemed to have cirrhosis if preprocedural clinical evaluation, laboratory tests, imaging studies, and endoscopic examination were consistent or suggestive of PH. Measurements were conducted in the PV and the HV. The inferior vena cava (IVC) was targeted when HV was inaccessible because of anatomic limitations. Technical success was defined as a successful PPG measurement in each patient. Universal definitions of $\mathrm{PH}(>5 \mathrm{~mm} \mathrm{Hg})$ and $\mathrm{CSPH}$ ( $>10 \mathrm{~mm} \mathrm{Hg}$ ) were used. Technical success was achieved in all 28 (100\%) patients. Fifteen of 28 (57.1\%) patients had evidence of PH based on PPG, of whom 10 of 15 (66.7\%) patients had CSPH. Eleven of 28 subjects had endoscopic evidence of either esophageal or gastric varices. All 11 (100\%) patients with endoscopic evidence of varices had $\mathrm{PH}$ and $10(90.9 \%)$ patients had CSPH. In 9 (32.1\%) patients, access to HV was difficult due to anatomic distortion; in these patients, IVC pressure measurements were taken. Importantly, despite enrolling patients with some degree of thrombocytopenia and underlying cirrhosis, there were no intraprocedural or postprocedural adverse events and no infection.

PPG levels were increased in those with high clinical evidence of cirrhosis and in those with varices, Portal Hypertensive Gastropathy (PHG), and thrombocytopenia, compared with those without these conditions. The mean PPGs were 8.5 and $3.5 \mathrm{~mm} \mathrm{Hg}$ for patients with and without high evidence for cirrhosis, 13.8 and $3.9 \mathrm{~mm} \mathrm{Hg}$ with and without varices, and 11.9 and $4.8 \mathrm{~mm} \mathrm{Hg}$ with and without PHG, respectively. Per logistic regression analysis, in a patient with PPG $>5 \mathrm{~mm} \mathrm{Hg}$, the odds of high evidence of cirrhosis were 18.7-fold higher than a patient with a normal measurement ( $<5 \mathrm{~mm} \mathrm{Hg}$ ). When a patient has PPG $>5 \mathrm{~mm} \mathrm{Hg}$, the odds of having thrombocytopenia were 6.1 -fold higher than a patient with PPG $<5 \mathrm{~mm} \mathrm{Hg}$.

This study demonstrates the safety and feasibility of direct EUS-guided PPG measurement using a 25-G needle and a novel compact manometer in humans. The manometer used for PV pressure measurement is manufactured by Cook Medical. This device is a $25-G$ needle with a pressure sensor at the tip of the needle, which transmits the pressures in the vein to a manometer connected at the needle hub. The pressures are displayed in a digital manner. Medical management of $\mathrm{PH}$ currently involves $\beta$-blockers, which are tailored to achieve resting heart rate of less than 60 beats 
per minute. Incorporating direct PPG measurement in routine practice may help identify patients with inadequate control of portal pressure and thus assist in a tailored management approach for $\mathrm{PH}$. Also, newer therapies and their effects for PH management can be possible with this technique. Further, this has prognostic implications and Selected patients with very high portal pressures may be offered therapies such as Transjugular Intrahepatic PortoSystemic shunt (TIPS), if medical therapy is not effective. This technique will be especially useful in noncirrhotic $\mathrm{PH}$ patients in whom the WHVP does not accurately reflect the portal venous pressure and a direct pressure measurement is desirable. Overall, results of this study are promising and will enable a convenient approach for measurement of PPG, which can be an "add-on" to varices patients scheduled for upper GI endoscopy. Limitations of the study include retrospective study design. This study did not compare EUS-PPG with HVPG simultaneously, which limits validity of EUS-PPG. Also, deep sedation can have impact on PPG measurement, which needs to be addressed. Further studies are needed to establish the safety of this technique.

\section{Role of Rapid On-Site Evaluation for EUS-FNB in Solid Pancreatic Lesions}

EUS-guided tissue acquisition has been traditionally performed with the EUS-FNA needles. Diagnostic accuracy with EUS-FNA for solid pancreatic lesions is up to $91 \%{ }^{33}$ Supplementing EUS-FNA with rapid on-site evaluation (ROSE) has shown superior results compared with EUS-FNA alone. ${ }^{34-36}$ However, ROSE is not widely available, with the expertise largely limited to tertiary centers. More recently, several new EUS-guided fine needle biopsy (EUS-FNB) needles have been introduced with unique designs of needle tip providing superior tissue samples that provide material not only for cytology but also for histopathology (-Fig. 6). EUS-FNB has shown

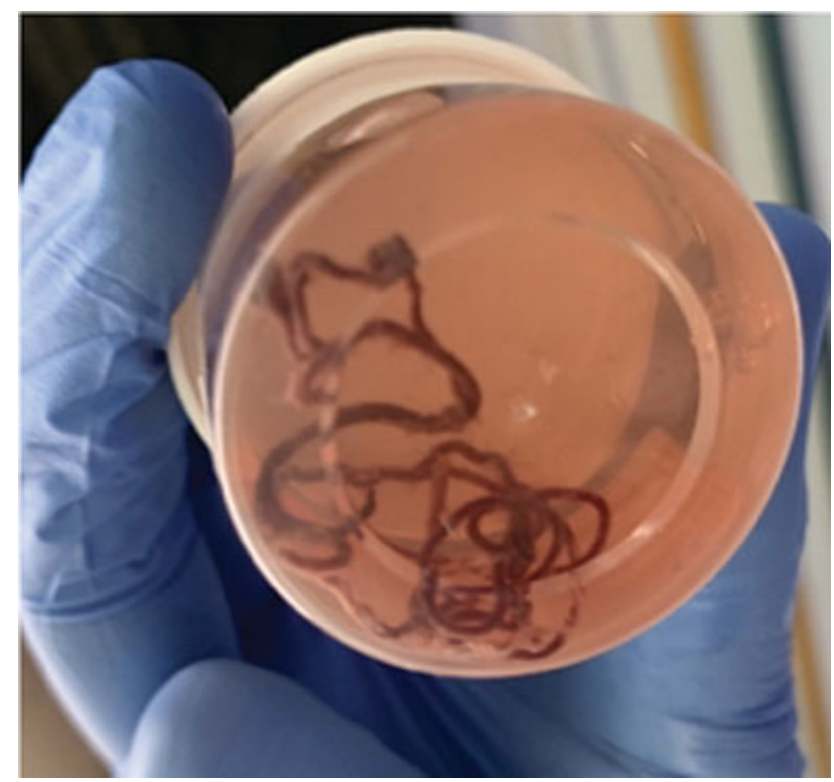

Fig. 6 Core specimen obtained with EUS-FNB. comparable results to EUS-FNA + ROSE. ${ }^{37}$ Chen et al showed that EUS-FNB is superior to EUS-FNA + ROSE in terms of diagnostic yield and is less time-consuming. ${ }^{38}$ While ROSE has benefit with EUS-FNA, its utility in patients undergoing EUS-FNB is not clear.EUS-guided tissue acquisition has been traditionally performed with the EUS-FNA needles. Diagnostic accuracy with EUS-FNA for solid pancreatic lesions is up to $91 \%{ }^{33}$ Supplementing EUS-FNA with rapid on-site evaluation (ROSE) has shown superior results compared with EUS-FNA alone. ${ }^{34-36}$ However, ROSE is not widely available, with the expertise largely limited to tertiary centers. More recently, several new EUS-guided fine needle biopsy (EUS-FNB) needles have been introduced with unique designs of needle tip providing superior tissue samples that provide material not only for cytology but also for histopathology (-Fig. 6). EUSFNB has shown comparable results to EUS-FNA + ROSE. ${ }^{37}$ Chen et al showed that EUS-FNB is superior to EUS-FNA + ROSE in terms of diagnostic yield and is less time-consuming. ${ }^{38}$ While ROSE has benefit with EUS-FNA, its utility in patients undergoing EUS-FNB is not clear.

Crinò et $\mathrm{al}^{39}$ performed a large international, multicenter, randomized, noninferiority, controlled trial comparing EUSFNB + ROSE versus EUS-FNB alone in solid pancreatic lesions in multiple centers in Italy. Majority of the evaluated patients were randomized (800 of 845 ) and only a small proportion were lost to follow-up ( 29 of 800 ). Procedures were performed by experienced endosonographers with any of the commonly used FNB needles (60\% Franseen-tip 22 G, 20\% fork-tip $22 \mathrm{G}$, and $20 \%$ side-fenestrated $20 \mathrm{G}$ ). Fanning was performed when possible ( $>90 \%$ in both arms) and tissue acquisition technique (slow-pull, suction) was at the discretion of the endosonographer, with most performing the slow-pull technique $(\sim 60 \%)$. There was no difference in distribution between the two groups based on the abovementioned variables. Three passes were performed in both the groups. In the ROSE arm, tissue imprint cytology was first performed by smearing the material on a slide with remainder of the material put in formalin, while in the EUS-FNBalone arm all the material was directly sent in formalin.

The study showed that the diagnostic accuracy was not different between the two arms-96\% in EUS-FNB + ROSE versus $97.3 \%$ in EUS-FNB alone. Majority of the patients had pancreatic adenocarcinoma as the final diagnosis (almost $80 \%$ ), and there was no difference in distribution of the diagnosis type between the two arms. Interestingly, core procurement was more in the EUS-FNB-alone arm (78 vs. $70 \%)$, likely related to loss of tissue in the EUS-FNB + ROSE arm for the purpose of tissue imprint cytology. There was a progressive increase in sample adequacy with ROSE-59.2\% first pass, $76.4 \%$ second pass, and $90.4 \%$ third pass. Final diagnosis could be achieved in $98.4 \%$-an additional $8 \%$ cases by supplementing with histopathological findings obtained from EUS-FNB. There were also some changes in interpretation of the results. In 41 patients with benign/atypical findings on ROSE, 60\% were upstaged to malignancy when the additional histopathologic specimen was reviewed. In one case, ROSE diagnosed the patient with malignancy but the histopathologic specimen downgrade to neuroendocrine 
Table 1 Advances in EUS and their impact on current clinical care

\begin{tabular}{|l|l|}
\hline Advances in EUS & Impact on current clinical care \\
\hline EUS-GBD & $\begin{array}{l}\text { EUS-GBD is superior to PT-GBD in high-risk cholecystitis patients. It is also } \\
\text { helpful with high technical and clinical success for malignant biliary } \\
\text { obstruction with failed ERCP and EUS-BD }\end{array}$ \\
\hline $\begin{array}{l}\text { EUS-guided management of } \\
\text { infected pancreatic necrosis }\end{array}$ & $\begin{array}{l}\text { EUS-guided management is associated with less morbidity and mortality as } \\
\text { compared with surgical or minimal invasive surgical approach }\end{array}$ \\
\hline EUS-guided PPG measurement & $\begin{array}{l}\text { It is safe and feasible. Also, it has advantages over HVPG measurement in } \\
\text { terms of radiation exposure. Patients posted for upper gastrointestinal } \\
\text { endoscopy can undergo PPG measurement in the same setting, which helps } \\
\text { determine the pharmacotherapy, its dose, and prognosis }\end{array}$ \\
\hline $\begin{array}{l}\text { EUS-guided FNB for solid } \\
\text { pancreatic lesions }\end{array}$ & $\begin{array}{l}\text { There is no difference in tissue acquisition by addition of ROSE to EUS-FNB, } \\
\text { thus obviating the need for ROSE when using FNB needles. EUS-FNB without } \\
\text { ROSE is shorter and convenient, enabling high tissue acquisition rates even in } \\
\text { community setting where ROSE is not widely available }\end{array}$ \\
\hline
\end{tabular}

Abbreviations: ERCP, endoscopic retrograde cholangiopancreatography; EUS-BD, endoscopic ultrasound-guided bile duct drainage; EUS-FNB, endoscopic ultrasound-guided fine needle biopsy; EUS-GBD, endoscopic ultrasound-guided gall bladder drainage; HVPG, hepatic venous pressure gradient; PPG, portal pressure gradient; PT-GBD, percutaneous gall bladder drainage; ROSE, rapid on-site evaluation.

tumor, thus underscoring the importance of evaluation of the core specimen, which provided valuable information and changed the final diagnosis.

There was no difference in rate of adverse events-2.1\% in EUS-FNB + ROSE versus $1 \%$ in EUS-FNB alone. Importantly, procedure duration was much shorter in the EUS-FNB-alone arm compared with EUS-FNB + ROSE (11.7 vs. 17.9 minutes). This is expected as ROSE is time-consuming and requires staining and evaluation of the expressed material by a cytopathologist, thus prolonging the procedure. In a side analysis of comparison of the needles, the front-edge cutting $22-G$ needles had a trend toward better performance with higher core procurement and lower blood contamination, without any difference in adverse events compared with the $20-G$ side-fenestrated needle. However, the limitation of this study is that they used three different needles without randomization, which could have affected the result.

This study validates the high diagnostic accuracy of EUS-FNB ( $>95 \%$ ) and demonstrates no added advantage of ROSE for patients undergoing tissue acquisition for solid pancreatic lesions, thus obviating the need for ROSE while performing EUS-FNB for solid pancreatic lesions. Additionally, the procedure duration was shorter with EUS-FNB-alone group, which can lead to higher efficiency for busy centers and cost-savings overall. Whether these results can be applied to other masses such as lymph nodes and subepithelial lesions remains to be seen.

\section{Summary}

The current article discusses four important studies that in our opinion will impact the role of EUS in managing various conditions (-Table 1). For patients with acute cholecystitis, EUS-GBD is safe and superior to PT-GBD in terms of reinterventions, pain scores, and hospital stay. Endoscopic transmural management of acute necrotizing pancreatitis has better outcome than minimally invasive surgery in terms of hospital stay, morbidity, and mortality. In patients with
PH, EUS-guided PPG measurement is safe and feasible. EUSFNB + ROSE has no added advantage over EUS-FNB alone. Thus, without ROSE, excellent tissue specimens can be retrieved with EUS-FNB alone.

\section{Conflict of Interest \\ None declared.}

\section{References}

1 Okamoto K, Suzuki K, Takada T, et al. Tokyo Guidelines 2018: flowchart for the management of acute cholecystitis. J Hepatobiliary Pancreat Sci 2018;25(01):55-72

2 Chopra S, Dodd GD III, Mumbower AL, et al. Treatment of acute cholecystitis in non-critically ill patients at high surgical risk: comparison of clinical outcomes after gallbladder aspiration and after percutaneous cholecystostomy. AJR Am J Roentgenol 2001; 176(04):1025-1031

3 Winbladh A, Gullstrand P, Svanvik J, Sandström P. Systematic review of cholecystostomy as a treatment option in acute cholecystitis. HPB (Oxford) 2009;11(03):183-193

4 Sanjay P, Mittapalli D, Marioud A, White RD, Ram R, Alijani A. Clinical outcomes of a percutaneous cholecystostomy for acute cholecystitis: a multicentre analysis. HPB (Oxford) 2013;15(07): 511-516

5 Itoi T, Sofuni A, Itokawa F, et al. Endoscopic transpapillary gallbladder drainage in patients with acute cholecystitis in whom percutaneous transhepatic approach is contraindicated or anatomically impossible (with video). Gastrointest Endosc 2008;68(03):455-460

6 Tyberg A, Saumoy M, Sequeiros EV, et al. EUS-guided versus percutaneous gallbladder drainage: isn't it time to convert? J Clin Gastroenterol 2018;52(01):79-84

7 Irani S, Ngamruengphong S, Teoh A, et al. Similar efficacies of endoscopic ultrasound gallbladder drainage with a lumen-apposing metal stent versus percutaneous transhepatic gallbladder drainage for acute cholecystitis. Clin Gastroenterol Hepatol 2017;15(05):738-745

8 Teoh AYB, Serna C, Penas I, et al. Endoscopic ultrasound-guided gallbladder drainage reduces adverse events compared with percutaneous cholecystostomy in patients who are unfit for cholecystectomy. Endoscopy 2017;49(02):130-138 
9 Teoh AYB, Kitano M, Itoi T, et al. Endosonography-guided gallbladder drainage versus percutaneous cholecystostomy in very high-risk surgical patients with acute cholecystitis: an international randomised multicentre controlled superiority trial (DRAC 1). Gut 2020;69(06):1085-1091

10 Kedia P, Sharaiha RZ, Kumta NA, et al. Endoscopic gallbladder drainage compared with percutaneous drainage. Gastrointest Endosc 2015;82(06):1031-1036

11 Bang JY, Arnoletti JP, Holt BA, et al. An endoscopic transluminal approach, compared with minimally invasive surgery, reduces complications and costs for patients with necrotizing pancreatitis. Gastroenterology 2019;156(04):1027-1040.e3

12 Lisotti A, Cominardi A, Bacchilega I, Linguerri R, Fusaroli P. EUSguided transrectal drainage of pelvic fluid collections using electrocautery-enhanced lumen-apposing metal stents: a case series. VideoGIE 2020;5(08):380-385

13 Saumoy M, Tyberg A, Brown E, et al. Successful cholecystectomy after endoscopic ultrasound gallbladder drainage compared with percutaneous cholecystostomy, can it be done? J Clin Gastroenterol 2019;53(03):231-235

14 Issa D, Irani S, Law R, et al. Endoscopic ultrasound-guided gallbladder drainage as a rescue therapy for unresectable malignant biliary obstruction: a multicenter experience. Endoscopy 2021;53(08):827-831

15 Tenner S, Baillie J, DeWitt J, Vege SSAmerican College of Gastroenterology. American College of Gastroenterology guideline: management of acute pancreatitis. Am J Gastroenterol 2013; 108(09):1400-1415; 1416

16 Whitcomb DC. Clinical practice. Acute pancreatitis. N Engl J Med 2006;354(20):2142-2150

17 van Santvoort HC, Besselink MG, Bakker OJ, et al; Dutch Pancreatitis Study Group. A step-up approach or open necrosectomy for necrotizing pancreatitis. N Engl J Med 2010;362(16):1491-1502

18 Bakker OJ, van Santvoort HC, van Brunschot S, et al; Dutch Pancreatitis Study Group. Endoscopic transgastric vs surgical necrosectomy for infected necrotizing pancreatitis: a randomized trial. JAMA 2012;307(10):1053-1061

19 van Brunschot S, van Grinsven J, Voermans RP, et al; Dutch Pancreatitis Study Group. Transluminal endoscopic step-up approach versus minimally invasive surgical step-up approach in patients with infected necrotising pancreatitis (TENSION trial): design and rationale of a randomised controlled multicenter trial [ISRCTN09186711]. BMC Gastroenterol 2013;13:161

20 Singh VK, Wu BU, Bollen TL, et al. Early systemic inflammatory response syndrome is associated with severe acute pancreatitis. Clin Gastroenterol Hepatol 2009;7(11):1247-1251

21 Strøm T, Martinussen T, Toft P. A protocol of no sedation for critically ill patients receiving mechanical ventilation: a randomised trial. Lancet 2010;375(9713):475-480

22 Bosch J, Abraldes JG, Groszmann R. Current management of portal hypertension. J Hepatol 2003;38(Suppl 1):S54-S68

23 Sanyal AJ, Bosch J, Blei A, Arroyo V. Portal hypertension and its complications. Gastroenterology 2008;134(06):1715-1728

24 Burroughs AK, McCormick PA. Natural history and prognosis of variceal bleeding. Baillieres Clin Gastroenterol 1992;6(03):437-450

25 D'Amico G, Garcia-Tsao G, Pagliaro L. Natural history and prognostic indicators of survival in cirrhosis: a systematic review of 118 studies. J Hepatol 2006;44(01):217-231
26 Villanueva C, Miñana J, Ortiz J, et al. Endoscopic ligation compared with combined treatment with nadolol and isosorbide mononitrate to prevent recurrent variceal bleeding. N Engl J Med 2001; 345(09):647-655

27 Villanueva C, Balanzó J, Novella MT, et al. Nadolol plus isosorbide mononitrate compared with sclerotherapy for the prevention of variceal rebleeding. N Engl J Med 1996;334(25):1624-1629

28 Feu F, García-Pagán JC, Bosch J, et al. Relation between portal pressure response to pharmacotherapy and risk of recurrent variceal haemorrhage in patients with cirrhosis. Lancet 1995; 346(8982):1056-1059

29 Armonis A, Patch D, Burroughs A. Hepatic venous pressure measurement: an old test as a new prognostic marker in cirrhosis? Hepatology 1997;25(01):245-248

30 Huang JY, Samarasena JB, Tsujino T, Chang KJ. EUS-guided portal pressure gradient measurement with a novel 25-gauge needle device versus standard transjugular approach: a comparison animal study. Gastrointest Endosc 2016;84(02):358-362

31 Zhang W, Peng C, Zhang S, et al. EUS-guided portal pressure gradient measurement in patients with acute or subacute portal hypertension. Gastrointest Endosc 2021;93(03):565-572

32 Huang JY, Samarasena JB, Tsujino T, et al. EUS-guided portal pressure gradient measurement with a simple novel device: a human pilot study. Gastrointest Endosc 2017;85(05):996-1001

33 Banafea O, Mghanga FP, Zhao J, Zhao R, Zhu L. Endoscopic ultrasonography with fine-needle aspiration for histological diagnosis of solid pancreatic masses: a meta-analysis of diagnostic accuracy studies. BMC Gastroenterol 2016;16:108

34 Hébert-Magee S, Bae S, Varadarajulu S, et al. The presence of a cytopathologist increases the diagnostic accuracy of endoscopic ultrasound-guided fine needle aspiration cytology for pancreatic adenocarcinoma: a meta-analysis. Cytopathology 2013;24(03): 159-171

35 Matynia AP, Schmidt RL, Barraza G, Layfield LJ, Siddiqui AA, Adler DG. Impact of rapid on-site evaluation on the adequacy of endoscopic-ultrasound guided fine-needle aspiration of solid pancreatic lesions: a systematic review and meta-analysis. J Gastroenterol Hepatol 2014;29(04):697-705

36 Iglesias-Garcia J, Dominguez-Munoz JE, Abdulkader I, et al. Influence of on-site cytopathology evaluation on the diagnostic accuracy of endoscopic ultrasound-guided fine needle aspiration (EUS-FNA) of solid pancreatic masses. Am J Gastroenterol 2011; 106(09):1705-1710

37 Khan MA, Grimm IS, Ali B, et al. A meta-analysis of endoscopic ultrasound-fine-needle aspiration compared to endoscopic ultrasound-fine-needle biopsy: diagnostic yield and the value of onsite cytopathological assessment. Endosc Int Open 2017;5(05): E363-E375

38 Chen YI, Chatterjee A, Berger R, et al. Endoscopic ultrasound (EUS)-guided fine needle biopsy alone vs. EUS-guided fine needle aspiration with rapid onsite evaluation in pancreatic lesions: a multicenter randomized trial. Endoscopy 2021 (e-pub ahead of print). 54(01):4-12. Doi: 10.1055/a-1375-9775

39 Crinò SF, Di Mitri R, Nguyen NQ et al. Endoscopic ultrasoundguided fine-needle biopsy with or without rapid on-site evaluation for diagnosis of solid pancreatic lesions: a randomized controlled non-inferiority trial. Gastroenterology 2021;161 (03):899-909.e5 